\title{
Combined Impact of Positive Screen for Sarcopenia and Frailty on Physical Function, Cognition and Nutrition in the Community Dwelling Older Adult
}

\author{
Hsien Xiong Lee ${ }^{1}$, Audrey $\mathrm{Yeo}^{2}$, Cai Ning Tan ${ }^{2}$, Suzanne Yew ${ }^{2}$, Laura Tay ${ }^{3}$, Yew Yoong Ding ${ }^{2,4}$, Wee Shiong Lim ${ }^{2,4}$ \\ ${ }^{1}$ Department of Geriatric Medicine, Woodlands Health Campus, Singapore \\ ${ }^{2}$ Institute of Geriatrics and Active Ageing, Tan Tock Seng Hospital, Singapore \\ ${ }^{3}$ Department of Internal Medicine, Sengkang Hospital, Singapore \\ ${ }^{4}$ Department of Geriatric Medicine, Tan Tock Seng Hospital, Singapore
}

Corresponding Author:

Hsien Xiong Lee, MRCP, MMed

Department of Geriatric Medicine,

Woodlands Health Compus, 2 Yishun

Central 2, Singapore 768024

E-mail: Raphael_lee@whc.sg

ORCID:

https://orcid.org/0000-0001-6125-1100

Received: June 24, 2021

Revised: September 7, 2021

Accepted: September 7, 2021
Background: While sarcopenia and frailty independently contribute to functional impairment and disability, the combined impact resulting from their interplay is unclear. We investigated if functional, physical, cognitive, and nutritional measures were more adversely affected in community-dwelling older adults who were screened positive for both frailty and sarcopenia. Methods: Using the FRAIL $(\geq 1)$ and SARC-F (Strength, Assistance with walking, Rising from a chair, Climbing stairs, and Falls) ( $\geq 1$ ) scales for screening, we categorized 200 participants (age, $67.9 \pm 7.9$ years) as combined (both positive, 12.5\%), intermediate (either positive, 25.5\%), or robust (both negative, 62\%). Results: Comparisons of the three groups showed that the combined group had significantly worse functional ability (Frenchay Activities Index and Modified Barthel Index), physical performance (knee extension, gait speed, and Short Physical Performance Battery score), cognition/mood (Chinese Mini-Mental State Examination [CMMSE] score and Geriatric Depression Scale), and nutrition (Mini Nutritional Assessment [MNA] score) ( $p<0.05$, one-way analysis of variance). Post-hoc comparisons revealed similar findings between the combined and robust groups, except for knee extension and CMMSE scores. Only MNA scores were significantly lower between the intermediate and robust groups. Conclusion: Functional ability, physical performance, and nutrition were more adversely affected in our study population of community-dwelling older adults who screened positive for both frailty and sarcopenia than in those who screened positive for either or neither, supporting the use of community screening for early detection and intervention for both frailty and sarcopenia as opposed to either alone.

Key Words: Sarcopenia, Frailty, Community-dwelling

\section{INTRODUCTION}

Frailty is a modern geriatric giant, ${ }^{1,2)}$ defined as a state of increased vulnerability with a poor restoration of homeostasis after a stressor event that increases an individual's susceptibility to increased dependency, adverse outcomes, and death. ${ }^{3-5)}$ The reported prevalence of frailty in community-dwelling older adults in the Asia-Pacific region is $3.5 \%-27 \%{ }^{2)}$ With the worldwide demographic trend of population aging, the impact of frailty is expected to rise in tandem, resulting in an increasing burden on healthcare systems and the escalation of healthcare costs. ${ }^{6,7)}$

Sarcopenia is a related but a distinct condition ${ }^{8)}$ that refers to the progressive loss of skeletal muscle mass and strength that occurs with aging." Similar to frailty, sarcopenia predisposes older individuals to adverse consequences such as falls, disability, and mortality. ${ }^{9,10)}$ The reported prevalence of sarcopenia is $6 \%-22 \%$ in adults aged $\geq 65$ years, depending on the care setting. ${ }^{11)}$ The plausible biological mechanisms linking sarcopenia and frailty include 
inflammatory responses, oxidative stress, and hormonal dysregulation. $^{10,12)}$ The complex intertwining relationship between sarcopenia and frailty has been likened to a "chicken and egg" situation. Sarcopenia is believed to be a fundamental component and possible antecedent of frailty, resulting in reduced muscle strength and gait speed, which characterize the physical manifestation of frailty. ${ }^{12-15)}$ Conversely, weight loss, sedentary behavior, cognitive impairment, and social isolation, which are commonly associated with frailty, can lead to reduced muscle mass and impaired muscle function. ${ }^{14,16)}$

While sarcopenia and frailty independently contribute to functional impairment and disability, the combined impact resulting from the interplay between these two conditions is unclear. Sarcopenia and frailty have generally been studied separately in isolation rather than in parallel. ${ }^{14,15)}$ Using the Edmonton Frail Scale and the SARC-F (Strength, Assistance with walking, Rising from a chair, Climbing stairs, and Falls) questionnaire, a study observed both frailty and sarcopenia in approximately one-quarter of older adults attending medical specialist outpatient clinics. ${ }^{17)}$ The presence of both syndromes was associated with poorer self-rated health, recurrent hospital admissions, polypharmacy, multiple medical clinic appointments, higher fall rate, and increased falls with serious consequences. However, the study did not compare the combined effects of frailty and sarcopenia relative to the presence of either condition alone. It is also unclear whether these findings can be generalized to a population of more robust community-dwelling older adults.

Thus, we investigated whether functional, physical, cognitive, and nutritional measures were more adversely affected in community-dwelling older adults who were screened positive for both frailty and sarcopenia than in those who were screened positive for either condition alone. If true, this finding would support the use of community screening for the early identification of both conditions to facilitate early intervention and reduce poor health outcomes.

\section{MATERIALS AND METHODS}

\section{Study Setting}

We studied 200 cognitively intact and functionally independent community-dwelling adults aged $\geq 50$ years who participated in the "Longitudinal Assessment of Biomarkers for characterization of early Sarcopenia and predicting frailty and functional decline in community-dwelling Asian older adults Study." The details of the study have been described previously. ${ }^{18)}$ In brief, the inclusion criteria included community-dwelling older adults aged 50-99 years who were cognitively intact and independent in both basic activi- ties of daily living (bADL) and instrumental ADL (iADL). The exclusion criteria were (1) presence of dementia or cognitive impairment-defined as a Chinese Mini-Mental State Score $($ CMMSE $) \leq 21{ }^{19)}(2)$ inability to walk at least $4.5 \mathrm{~m}$ independently, and (3) residing in nursing or sheltered homes. Informed written consent was obtained from the participants in the presence of a trained research assistant. This study was approved by the Institutional Review Board of the National Healthcare Group (NHG DSRB No. 2012/00897).

\section{Study Groups}

We used the five-item self-report FRAIL scale to screen for the presence of frailty. ${ }^{20)}$ The FRAIL contains five components: fatigue, resistance, ambulation, illnesses, and weight loss. The scores range from 0 to 5 and correspond to frail (3-5), pre-frail (1-2), and robust $(0)$ health status. Sarcopenia was screened using the five-item self-report SARC-F scale, which includes items related to slow speed while walking, assistance in walking, rising from a chair, climbing stairs, and falls. ${ }^{21)}$ The total scores range from 0 to 10 , with scores of $\geq 4$ indicating sarcopenia and adverse outcomes. Because our sample comprised fairly healthy community-dwelling older adults who were cognitively and functionally intact, ${ }^{22)}$ we used a FRAIL scale cut-off score of $\geq 1$ to identify pre-frail/frail participants. Similarly, we ascertained sarcopenia using a SARC-F scale cut-off score of $\geq 1$, which showed higher sensitivity and reasonable specificity for the detection of probable sarcopenia among community-dwelling older adults. ${ }^{23-25)}$ Using the aforementioned FRAIL and SARC-F scales cut-offs, we categorized the participants into three groups: (1) combined (positive for both frailty and sarcopenia), (2) intermediate (positive for either condition), and (3) robust (negative for both conditions).

\section{Data Collection}

We collected demographic data and information on vascular risk factors, including hypertension, hyperlipidemia, diabetes mellitus, atrial fibrillation, ischemic heart disease, stroke, transient ischemic attack, and smoking. We evaluated functional ability using the Modified Barthel Index (MBI) for bADL, ${ }^{26)}$ Lawton and Brody's iADL index, ${ }^{27)}$ and the Frenchay Activities Index as a measure of physical activity. ${ }^{28)}$ The MBI examines performance in bADL such as personal hygiene or grooming, dressing, toileting, transferring, or ambulation. The scores range from 0 to 100 , with higher scores indicating greater independence. The $\mathrm{iADL}$ index examines activities that allow for independent community living, such as the ability to use the telephone, shopping, food preparation, housekeeping, laundry, transportation, handling finances, and taking medications. The scores range from 0 to 8 , with higher 
scores indicating greater independence. The Frenchay Activities Index is complementary to the MBI by measuring higher-order activities of daily living, such as social and lifestyle activities. Broadly, they assess three subscales: domestic chores, leisure/ work, and outdoor activities. Scoring is based on the frequency with which 15 activities are performed, each of which is scored on a four-point scale ( 0 to 3 ) to yield a total score ranging from 0 (inactive) to 45 (active).

Regarding physical performance, we measured the upper and lower limb muscle strength and gait speed while walking a distance of $4.5 \mathrm{~m}$; we also used the Short Physical Performance Battery (SPPB). ${ }^{29)}$ Handgrip strength was measured using a hydraulic hand dynamometer (North Coast Medical Inc., Morgan Hill, CA, USA). Each subject had two readings of grip strength for each hand; we averaged all four readings to obtain a final value. Knee extension was measured using an electronic push/pull dynamometer (BASELINE 12-0342; Fabrication Enterprises Inc., White Plains, NY, USA). The participants sat with their legs over the edge of a chair with their hands resting on their thighs and their hips and knees flexed at $90^{\circ}$. The dynamometer was positioned immediately above the malleoli and perpendicular to the tibial crest with the monitor facing downward.

We assessed cognition using the CMMSE, ${ }^{19)}$ which was locally validated. We also assessed depressive symptoms using the 15 -item Geriatric Depression Scale. ${ }^{30)}$ Nutritional status was assessed in three ways: (1) body mass index (BMI), derived from measurements of standing height and weight of the participant; (2) Mini Nutritional Assessment (MNA) scale, which was locally validat$\mathrm{ed}^{31}{ }^{31}$ and (3) vitamin D level, with deficiency defined as a serum concentration of $<20 \mathrm{ng} / \mathrm{mL}$ based on modified Holick's classifications. $^{32)}$

\section{Statistical Analysis}

Descriptive data are presented as mean \pm standard deviatin or median (interquartile range, IQR) for quantitative variables and as absolute and relative frequencies for categorical variables. Inferential statistics were applied to compare differences in functional, cognitive, physical performance, and nutritional states between the three groups. We used one-way analysis of variance with Bonferroni correction for post-hoc comparisons; the Kruskal-Wallis test for parametric and non-parametric continuous variables; and the chisquare test for categorical variables. SPSS Statistics for Windows, version 23.0 (IBM, Armonk, NY, USA) was used for data analysis. All statistical tests were two-tailed, with the level of statistical significance set at $5 \%$.

\section{RESULTS}

\section{Baseline Characteristics}

Our study population comprised 200 older adults with a mean age of $67.9 \pm 7.9$ years, with female predominance $(68.5 \%)$, and mostly Chinese ethnicity (92\%). Using the FRAIL and SARC-F scales for screening, we identified 10 pre-frail/frail and 41 sarcopenic subjects. The robust, intermediate, and combined groups comprised 124 (62\%), 51 (25.5\%), and 25 (12.5\%) subjects, respectively. Age increased and educational level decreased moving from the robust to intermediate and combined groups. We observed no significant differences in sex, ethnicity, or cardiovascular risk factors. Not surprisingly, the combined group scored the highest on the FRAIL and SARC-F scales, followed by the intermediate and robust groups (both $\mathrm{p}<0.001)$ (Table 1$)$.

\section{Outcome Characteristics}

The combined group performed significantly worse in functional measures of bADL (100 [IQR 100-100] vs. 100 [IQR 100- 100] vs. 100 [IQR 95-100]; $\mathrm{p}=0.002$ ) and on the Frenchay Activities Index ( $32.77 \pm 4.50$ vs. $31.55 \pm 6.09$ vs. $29.80 \pm 5.24 ; \mathrm{p}=0.025)$, but not on $\mathrm{iADL}$, than other groups. For physical performance measures, the combined group had significantly worse SPPB scores (12 [IQR 11-12] vs. 12 [IQR 11-12] vs. 11 [IQR 8-12]; $\mathrm{p}=0.013)$, knee extension $(36.22 \pm 7.55$ vs. $33.63 \pm 7.88$ vs. $32.63 \pm 7.84 \mathrm{~kg} ; \mathrm{p}=0.031)$, and gait speed $(1.49 \pm 0.26$ vs. $1.49 \pm 0.26$ vs. $1.27 \pm 0.37 \mathrm{~m} / \mathrm{s} ; \mathrm{p}=0.01)$. We also observed a significant decrease in CMMSE scores from the robust to the intermediate/combined groups. Regarding nutritional measures, the MNA score was significantly lower in the intermediate and combined groups ( $27.40 \pm 1.77$ vs. $26.52 \pm 1.99$ vs. $25.98 \pm 2.33$; $\mathrm{p}=0.001$ ), but we observed no significant differences in vitamin $D$ levels and BMI. Post-hoc comparisons between the combined and robust groups revealed similar findings, except for knee extension and CMMSE scores. In contrast, only MNA scores were significantly lower in the post-hoc comparisons between the intermediate and robust groups (Table 2).

\section{DISCUSSION}

Conventionally, sarcopenia, being organ-specific, has been researched in the basic science domain, whereas frailty has been predominantly applied in clinical settings. ${ }^{14,33)}$ In the recent few years, their relationship has converged largely as a result of concerted efforts in recent consensus recommendations of sarcopenia to promote the translation of current knowledge into improved diagnosis and treatment in clinical practice. ${ }^{9)}$ Sarcopenia is now formally rec- 
Table 1. Baseline characteristics

\begin{tabular}{|c|c|c|c|c|}
\hline & Robust $(n=124)$ & Intermediate $(\mathrm{n}=51)$ & Combined $(n=25)$ & $\mathrm{p}$-value \\
\hline \multicolumn{5}{|l|}{ Demographics } \\
\hline Age $(y)$ & $67.35 \pm 7.77$ & $68.10 \pm 8.11$ & $70.52 \pm 7.57$ & 0.18 \\
\hline Sex, female & $83(67)$ & $34(67)$ & $20(80)$ & 0.42 \\
\hline Chinese ethnicity & $112(90)$ & $49(96)$ & $23(92)$ & 0.48 \\
\hline Education (y) & $9.81 \pm 4.51$ & $8.39 \pm 5.53$ & $6.48 \pm 4.17^{*}$ & 0.004 \\
\hline \multicolumn{5}{|l|}{ Cardiovascular risk factors } \\
\hline Diabetes & $22(18)$ & $13(26)$ & $8(32)$ & 0.21 \\
\hline Hypertension & $58(47)$ & $25(49)$ & $13(52)$ & 0.88 \\
\hline Hyperlipidemia & $85(69)$ & $30(59)$ & $17(68)$ & 0.46 \\
\hline Atrial fibrillation & $6(5)$ & $2(4)$ & $1(4)$ & 0.96 \\
\hline Ischemic heart disease & $3(2)$ & $0(0)$ & $1(4)$ & 0.44 \\
\hline Stroke/transient ischemic attack & $2(2)$ & $3(6)$ & $0(0)$ & 0.18 \\
\hline Smoking & $5(4)$ & $2(4)$ & $1(4)$ & 0.49 \\
\hline \multicolumn{5}{|l|}{ Frailty/sarcopenia } \\
\hline FRAIL score & $0(0-0)$ & $0(0-0)^{*}$ & $1(1-1)^{*}$ & $<0.001$ \\
\hline FRAIL $\geq 1$ & $0(0)$ & $10(20)^{*}$ & $25(100)^{*}$ & $<0.001$ \\
\hline SARC-F score & $0(0-0)$ & $1(1-1)^{*}$ & $2(1-2)^{*}$ & $<0.001$ \\
\hline SARC-F $\geq 1$ & $0(0)$ & $41(80)^{*}$ & $25(100)^{*}$ & $<0.001$ \\
\hline
\end{tabular}

Values are presented as mean \pm standard deviation or number (\%) or median (interquartile range).

${ }^{*} \mathrm{p}<0.01$, compared with the robust group (post-hoc test).

Table 2. Outcome characteristics

\begin{tabular}{|c|c|c|c|c|}
\hline & Robust $(n=124)$ & Intermediate $(\mathrm{n}=51)$ & Combined $(n=25)$ & $\mathrm{p}$-value \\
\hline \multicolumn{5}{|l|}{ Functional ability } \\
\hline bADL (0-100) & $100(100-100)$ & $100(100-100)$ & $100(95-100)^{*}$ & 0.002 \\
\hline iADL (0-23) & $23(23-23)$ & $23(23-23)$ & $23(23-23)$ & 0.089 \\
\hline FAI $(0-45)$ & $32.77 \pm 4.50$ & $31.55 \pm 6.09$ & $29.80 \pm 5.24^{* *}$ & 0.025 \\
\hline \multicolumn{5}{|l|}{ Physical performance } \\
\hline SPPB (0-12) & $12(11-12)$ & $12(11-12)$ & $11(8-12)^{*}$ & 0.013 \\
\hline Gait speed (m/s) & $1.49 \pm 0.26$ & $1.49 \pm 0.26$ & $1.27 \pm 0.37^{*}$ & 0.001 \\
\hline Knee extension (kg) & $36.22 \pm 7.55$ & $33.63 \pm 7.88$ & $32.63 \pm 7.84$ & 0.031 \\
\hline Grip strength (kg) & $22.44 \pm 6.70$ & $21.02 \pm 5.75$ & $19.57 \pm 6.70$ & 0.089 \\
\hline \multicolumn{5}{|l|}{ Cognition } \\
\hline CMMSE (0-28) & $26.44 \pm 1.66$ & $25.68 \pm 1.79$ & $25.84 \pm 1.84$ & 0.042 \\
\hline \multicolumn{5}{|l|}{ Mood } \\
\hline GDS (0-15) & $0(0-1)$ & $0(0-1)$ & $1(0-5)$ & 0.083 \\
\hline \multicolumn{5}{|l|}{ Nutritional measures } \\
\hline MNA (0-30) & $27.40 \pm 1.77$ & $26.52 \pm 1.99^{* *}$ & $25.98 \pm 2.33^{*}$ & 0.001 \\
\hline Vitamin D (ng/mL) & $29.64 \pm 9.52$ & $29.20 \pm 8.77$ & $28.25 \pm 11.90$ & 0.809 \\
\hline $\operatorname{BMI}\left(\mathrm{kg} / \mathrm{m}^{2}\right)$ & $23.94 \pm 3.54$ & $23.96 \pm 4.11$ & $24.16 \pm 4.34$ & 0.963 \\
\hline
\end{tabular}

Values are presented as median (interquartile range) or mean \pm standard deviation.

bADL, basic activities of daily living; iADL, instrumental activities of daily living; FAI, Frenchay Activities Index; SPPB, Short Physical Performance Battery; CMMSE, Chinese Mini-Mental State Examination; GDS, Geriatric Depression Scale; MNA, Mini-Nutrition Assessment; BMI, body mass index.

${ }^{*} \mathrm{p}<0.01,{ }^{* *} \mathrm{p}<0.05$, compared with the robust group (post-hoc test).

ognized as a muscle disease, with an International Classification of Diseases, Tenth Revision, Clinical Modification (ICD-10-CM) diagnosis code that can be used to bill for care in some countries. ${ }^{34)}$
Thus, instead of attempting to determine the precise clinical relationship between frailty and sarcopenia or to focus on each condition in isolation, ${ }^{35)}$ screening for the combination of both condi- 
tions may be a more pragmatic approach.

The results of our study support this approach in two ways. First, they demonstrate that the community proportion of older adults with both frailty and sarcopenia is not low. While the prevalence of $12.5 \%$ observed in our study is half of that reported in an earlier study of medical outpatients, ${ }^{17)}$ this could reflect differences in characteristics between study populations as well as the tools used to screen for or ascertain frailty. Second, our results affirmed that physical performance, $\mathrm{ADL}$, cognition, and nutrition were more adversely affected in our study population of community-dwelling older adults who screened positive for both frailty and sarcopenia. Notably, the combined effect of both syndromes on adverse outcomes was greater than that of either condition alone.

It is interesting to consider the theoretical framework that underpins this observation. Consistent reports of sarcopenia as opposed to the converse situation of sarcopenia with concomitant frailty in frail older adults supports the premise that sarcopenia may precede physical frailty. ${ }^{8,17)}$ Nonetheless, there lies a subset of older adults who are frail but not sarcopenic, presumably owing to other non-physical mechanisms of frailty such as cognitive impairment, mood disorders, and social isolation. ${ }^{14,36)}$ The combined presence of sarcopenia and frailty may thus identify the subset of older adults (1) with a more severe degree of sarcopenia with the onset of concomitant frailty or (2) who are initially frail not owing to physical reasons but who subsequently go on to develop physical consequences of frailty with resultant sarcopenia. This line of reasoning is supported by the latest European Working Group on Sarcopenia in Older People consensus. ${ }^{37)}$ Physical performance measures such as gait speed and SPPB, which overlap with the physical phenotype of frailty, are also used to define the severity of sarcopenia, corroborating concomitant frailty and the association with more severe sarcopenia.

Another finding was the lower CMMSE scores in the intermediate/combined group. A recent meta-analysis showed that sarcopenia was independently associated with cognitive impairment. ${ }^{38)}$ Moreover, sarcopenia-related mechanisms such as oxidative stress, inflammation, and insulin resistance can lead concurrently to vascular aging and neuronal dysfunction that, in turn, precipitate cognitive impairment with a risk of dementia. ${ }^{10,39)}$ Additionally, there is increasing appreciation for "cognitive frailty," in which physical frailty co-exists with cognitive impairment and results in an increased risk of functional decline and dementia. ${ }^{36)}$ Similarly, the association between the combined group and worse nutritional status as measured using MNA scores may be attributable either to nutritional factors linked to sarcopenia/frailty, notably vitamin D and insufficient protein intake, or conversely, the resultant functional impairment from sarcopenia/frailty, which may impact food access and preparation, causing nutritional deficits. ${ }^{40)}$

Taken together, our study results add to the growing body of evidence regarding the intertwined relationship between sarcopenia and frailty by corroborating the deleterious impact of the combination of sarcopenia and frailty on functional, physical, cognitive, and nutritional domains beyond those for either condition alone. ${ }^{35)}$ These findings support the use of a community screening strategy based on the FRAIL and SARC-F scales for the early identification of the combination of conditions (as opposed to either alone) to facilitate early intervention and reduce poor health outcomes, especially in at-risk groups such as older adults with diabetes and cognitive symptoms. A recent study on persons with diabetes mellitus reported that $42.5 \%$ of sarcopenic subjects were frail or pre-frail $^{39)}$ and that individuals with both conditions had an increased risk of adverse outcomes. ${ }^{41)}$ Similarly, two-thirds of individuals with mild cognitive impairment were physically frail or prefrail, which was closely associated with physical and functional impairments. ${ }^{41)}$ Thus, the early identification in at-risk groups followed by the timely institution of evidence-based interventions to address sarcopenia and frailty such as improving protein and calorie intake, prescribing exercise programs with resistive components, addressing polypharmacy, and treating vitamin $\mathrm{D}$ deficiency may help avert downstream deleterious consequences such as falls, disability, and institutionalization. ${ }^{2,11,42)}$

This study had some limitations. For instance, we included community-dwelling older adults who were functionally independent and had high baseline scores on the functional assessment scale. As such, small differences in function may not be detected by these scales owing to the ceiling effect. This limitation probably accounted for the lack of differences in iADL scores among the three groups. ${ }^{43)}$ As an exploratory cross-sectional study, it could determine associations but could not infer causality as temporality was not known. Analysis of the 2-year follow-up data of our cohort will demonstrate whether these findings hold true in longitudinal follow-ups. Our results, based on a cohort of functionally well community-dwelling older adults, may not be readily generalizable to other settings with more heterogeneous populations of older adults. In addition, the small sample size precluded further subgroup analysis of the independent effects of sarcopenia or frailty in the intermediate group. Hence, the emphasis of our study was on the comparison of outcomes among the combined, intermediate, and robust groups. Future studies are needed to examine the trajectory of changes in blood biomarkers that may be involved in the underlying pathogenesis before and after its development to delineate the relationship between sarcopenia and frailty in the combined group.

In conclusion, functional ability, physical performance, and nu- 
trition were more adversely affected in our study population of community-dwelling older adults who screened positive for both frailty and sarcopenia than in those positive for either or neither condition. Our findings support screening for both sarcopenia and frailty among community-dwelling adults to effect interventional measures to preserve function and avoid disability.

\section{ACKNOWLEDGMENTS}

\section{CONFLICT OF INTEREST}

The researchers claim no conflicts of interest.

\section{FUNDING}

This study was funded by a 2013 Lee Foundation Grant. We thank the following Senior Activity Centers (SACs): Wesley SAC, Care Corner SAC, Xin Yuan Community Service, Potong Pasir Wellness Centre, Tung Ling Community Services (Marine Parade and Bukit Timah), Viriya Community Services-My Centre@ Moulmein, House of Joy) and the study participants who have graciously consented to participate in the study.

\section{AUTHOR CONTRIBUTIONS}

Conceptualization, WSL, YYD, LT; Data Curation, AY, CNT, SY; Formal Analysis, WSL, HXL, CNT; Funding Acquisition, WSL, YYD, Methodology, WSL, YYD; Project Administration, WSL; Supervision, WSL; Visualization, WSL, HXL, Writing-original draft, WSL, YYD, HXL; Writing-review and editing, WSL, YYD, HXL.

\section{REFERENCES}

1. Morley JE, Vellas B, van Kan GA, Anker SD, Bauer JM, Bernabei R, et al. Frailty consensus: a call to action. J Am Med Dir Assoc 2013;14:392-7.

2. Dent E, Lien C, Lim WS, Wong WC, Wong CH, Ng TP, et al. The Asia-Pacific Clinical Practice Guidelines for the management of frailty. J Am Med Dir Assoc 2017; 18:564-75.

3. Clegg A, Young J, Iliffe S, Rikkert MO, Rockwood K. Frailty in elderly people. Lancet 2013;381:752-62.

4. Chong E, Ho E, Baldevarona-Llego J, Chan M, Wu L, Tay L, et al. Frailty in hospitalized older adults: comparing different frailty measures in predicting short- and long-term patient outcomes. J Am Med Dir Assoc 2018; 19:450-7.

5. Chew J, Lim WS, Chong MS, Ding YY, Tay L. Impact of frailty and residual subsyndromal delirium on 1-year functional recovery: a prospective cohort study. Geriatr Gerontol Int 2017;17: 2472-8.
6. Lim WS, Wong SF, Leong I, Choo P, Pang WS. Forging a frailty-ready healthcare system to meet population ageing. Int J Environ Res Public Health 2017;14:1448.

7. Lim WS, Wong CH, Ding YY, Rockwood K, Lien C. Translating the science of frailty in Singapore: results from the National Frailty Consensus Discussion. Ann Acad Med Singap 2019; 48:25-31.

8. Davies B, Garcia F, Ara I, Artalejo FR, Rodriguez-Manas L, Walter S. Relationship between sarcopenia and frailty in the Toledo study of healthy aging: a population based cross-sectional study. J Am Med Dir Assoc 2018; 19:282-6.

9. Chen LK, Liu LK, Woo J, Assantachai P, Auyeung TW, Bahyah KS, et al. Sarcopenia in Asia: consensus report of the Asian Working Group for Sarcopenia. J Am Med Dir Assoc 2014;15: 95-101.

10. Yu Z, Ruan Q D’Onofrio G, Greco A. From sarcopenia to frailty: the pathophysiological basis and potential target molecules of intervention. In: Dionyssiotis Y, editor. Frailty and sarcopenia: onset, development and clinical challenges. Rijeka, Croatia: InTech; 2017.p. 55-69.

11. Dent E, Morley JE, Cruz-Jentoft AJ, Arai H, Kritchevsky SB, Guralnik J, et al. International Clinical Practice Guidelines for Sarcopenia (ICFSR): screening, diagnosis and management. J Nutr Health Aging 2018;22:1148-61.

12. Cesari M, Pahor M, Lauretani F, Zamboni V, Bandinelli S, Bernabei R, et al. Skeletal muscle and mortality results from the InCHIANTI Study. J Gerontol A Biol Sci Med Sci 2009;64:37784.

13. Fried LP, Tangen CM, Walston J, Newman AB, Hirsch C, Gottdiener J, et al. Frailty in older adults: evidence for a phenotype. J Gerontol A Biol Sci Med Sci 2001;56:M146-56.

14. Cesari M, Landi F, Vellas B, Bernabei R, Marzetti E. Sarcopenia and physical frailty: two sides of the same coin. Front Aging Neurosci 2014;6:192.

15. Landi F, Cherubini A, Cesari M, Calvani R, Tosato M, Sisto A, et al. Sarcopenia and frailty: from theoretical approach into clinical practice. Eur Geriatr Med 2016;7:197-200.

16. Pek K, Chew J, Lim JP, Yew S, Tan CN, Yeo A, et al. Social frailty is independently associated with mood, nutrition, physical performance, and physical activity: insights from a theory-guided approach. Int J Environ Res Public Health 2020;17:4239.

17. Tan LF, Lim ZY, Choe R, Seetharaman S, Merchant R. Screening for frailty and sarcopenia among older persons in medical outpatient clinics and its associations with healthcare burden. J Am Med Dir Assoc 2017;18:583-7.

18. Tay L, Ding YY, Leung BP, Ismail NH, Yeo A, Yew S, et al. Sex-specific differences in risk factors for sarcopenia amongst 
community-dwelling older adults. Age (Dordr) 2015;37:121.

19. Sahadevan S, Lim PP, Tan NJ, Chan SP. Diagnostic performance of two mental status tests in the older chinese: influence of education and age on cut-off values. Int J Geriatr Psychiatry 2000; 15:234-41.

20. Morley JE, Malmstrom TK, Miller DK. A simple frailty questionnaire (FRAIL) predicts outcomes in middle aged African Americans. J Nutr Health Aging 2012;16:601-8.

21. Malmstrom TK, Miller DK, Simonsick EM, Ferrucci L, Morley JE. SARC-F: a symptom score to predict persons with sarcopenia at risk for poor functional outcomes. J Cachexia Sarcopenia Muscle 2016;7:28-36.

22. Lim WS, Tay L, Yeo A, Yew S, Hafizah N, Ding YY. Modulating effect of contextual factors on factor structure and reliability of SARC-F. J Am Med Dir Assoc 2018; 19:551-3.

23. Dodds RM, Murray JC, Robinson SM, Sayer AA. The identification of probable sarcopenia in early old age based on the SARC-F tool and clinical suspicion: findings from the 1946 British birth cohort. Eur Geriatr Med 2020;11:433-41.

24. Erbas Sacar D, Kilic C, Karan MA, Bahat G. Ability of SARC-F to find probable sarcopenia cases in older adults. J Nutr Health Aging 2021;25:757-61.

25. Lim WS, Tay L, Yeo A, Yew S, Hafizah N, Ding YY. SARC-F: defining a validated cutoff for pre-sarcopenia for risk assessment among community dwelling older persons. Australas J Ageing 2018;37(S1):41.

26. Mahoney FI, Barthel DW. Functional evaluation: the Barthel Index. Md State Med J 1965;14:61-5.

27. Barberger-Gateau P, Commenges D, Gagnon M, Letenneur L, Sauvel C, Dartigues JF. Instrumental activities of daily living as a screening tool for cognitive impairment and dementia in elderly community dwellers. J Am Geriatr Soc 1992;40:1129-34.

28. Wade DT, Legh-Smith J, Langton Hewer R. Social activities after stroke: measurement and natural history using the Frenchay Activities Index. Int Rehabil Med 1985;7:176-81.

29. Guralnik JM, Simonsick EM, Ferrucci L, Glynn RJ, Berkman LF, Blazer DG, et al. A short physical performance battery assessing lower extremity function: association with self-reported disability and prediction of mortality and nursing home admission. J Gerontol 1994;49:M85-94.

30. Yesavage JA, Brink TL, Rose TL, Lum O, Huang V, Adey M, et al. Development and validation of a geriatric depression screen- ing scale: a preliminary report. J Psychiatr Res 1982- 1983;17: 37-49.

31. Chan M, Lim YP, Ernest A, Tan TL. Nutritional assessment in an Asian nursing home and its association with mortality. J Nutr Health Aging 2010;14:23-8.

32. Thacher TD, Clarke BL. Vitamin D insufficiency. Mayo Clin Proc 2011;86:50-60.

33. Bauer JM, Sieber CC. Sarcopenia and frailty: a clinician's controversial point of view. Exp Gerontol 2008;43:674-8.

34. Anker SD, Morley JE, von Haehling S. Welcome to the ICD-10 code for sarcopenia. J Cachexia Sarcopenia Muscle 2016;7:5124.

35. Cruz-Jentoft AJ, Sayer AA. Sarcopenia. Lancet 2019;393:263646.

36. Kelaiditi E, Cesari M, Canevelli M, van Kan GA, Ousset PJ, Gillette-Guyonnet $\mathrm{S}$, et al. Cognitive frailty: rational and definition from an (I.A.N.A./I.A.G.G.) international consensus group. J Nutr Health Aging 2013;17:726-34.

37. Cruz-Jentoft AJ, Bahat G, Bauer J, Boirie Y, Bruyere O, Cederholm T, et al. Sarcopenia: revised European consensus on definition and diagnosis. Age Ageing 2019;48:16-31.

38. Chang KV, Hsu TH, Wu WT, Huang KC, Han DS. Association between sarcopenia and cognitive impairment: a systematic review and meta-analysis. J Am Med Dir Assoc 2016;17:1164.e71164.e15.

39. Liccini A, Malmstrom TK. Frailty and sarcopenia as predictors of adverse health outcomes in persons with diabetes mellitus. J Am Med Dir Assoc 2016;17:846-51.

40. Robinson S, Cooper C, Aihie Sayer A. Nutrition and sarcopenia: a review of the evidence and implications for preventive strategies.J Aging Res 2012;2012:510801.

41. Nyunt M, Soh CY, Gao Q, Gwee X, Ling A, Lim WS, et al. Characterisation of physical frailty and associated physical and functional impairments in mild cognitive impairment. Front Med (Lausanne) 2017;4:230.

42. Won CW. Frailty: its scope and implications for geriatricians. Ann Geriatr Med Res 2019;23:95-7.

43. Fieo RA, Austin EJ, Starr JM, Deary IJ. Calibrating ADL-IADL scales to improve measurement accuracy and to extend the disability construct into the preclinical range: a systematic review. BMC Geriatr 2011;11:42. 